\title{
Sindrome uremico-emolitica atipica: dalla patogenesi alla terapia
}

\author{
Selena Longhi, Giuseppe Pontoriero \\ S.C. Nefrologia e Dialisi ASST Lecco, Ospedale A. Manzoni, Lecco
}

\begin{abstract}
Atypical hemolytic-uremic syndrome: from pathogenesis to therapy
Thrombotic thrombocytopenic purpura and hemolytic uremic syndrome are part of a spectrum of thrombotic microangiopathies. In the last years enormous progress has been made in understanding the pathogenesis of these entities. The fact that complement dysregulation is considered central in the development of atypical hemolytic uremic syndrome has led to the introduction of Eculizumab, a monoclonal anti-C5 inhibitor, in its treatment. These new advances have improved the outcomes in patients with atypical hemolytic uremic syndrome. Many unsolved issues remains, the most pressing ones being identifying the optimal treatment schedules and the underlying cause of the disease in patients without detectable complement mutations.
\end{abstract}

Keywords: Atypical hemolytic uremic syndrome, Complement, Eculizumab

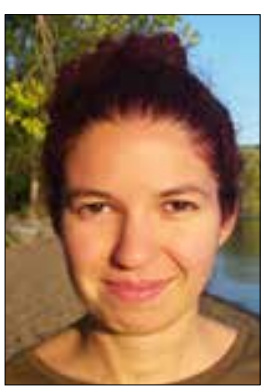

Selena Longhi

\section{Introduzione}

La sindrome uremico-emolitica (SEU) e la porpora trombotica trombocitopenica (TTP) rappresentano le due principali entità patologiche responsabili dello sviluppo delle più classiche forme di microangiopatia trombotica (TMA). L'identificazione del ruolo chiave giocato dall'ADAMTS 13 nella patogenesi della TTP e dalla disregolazione della via alternativa del complemento in quella della SEU ha portato a una migliore definizione eziopatogenetica di queste forme di microangiopatie, consentendo lo sviluppo di terapie mirate in grado di migliorare l'outcome dei pazienti. Sotto il termine SEU sono raggruppate le forme tipiche (o post-diarrea) dovute all'infezione da E. Coli, produttore della tossina di Shiga e le forme secondarie ad alterazioni del complemento (sindrome uremico-emolitica atipica, aSEU). Nella nostra trattazione discuteremo di quest'ultima entità e, sulla

Accepted: July 17, 2016

Published online: July 28, 2016

Indirizzo per la corrispondenza:

Dr. ssa Selena Longhi

S.C. Nefrologia e Dialisi ASST Lecco

Ospedale A. Manzoni

23900 Lecco

selena.longhi@gmail.com base dei più recenti dati di letteratura, ci concentreremo sulle ultime scoperte in campo eziopatogenetico e sull'impiego dell'Eculizumab nel suo trattamento.

\section{Caratteristiche cliniche}

La aSEU può esordire in qualsiasi età, con maggiore frequenza durante il periodo infantile. Durante l'infanzia è ugualmente frequente in entrambi i generi, mentre l'esordio tardivo è più frequente nelle femmine (1).

Clinicamente, si presenta con la triade composta da anemia emolitica meccanica, trombocitopenia e coinvolgimento renale. La presenza di aptoglobina consumata, aumento delle LDH, test di Coombs negativo e schistociti allo striscio periferico conferma la microangiopatia con emolisi intravascolare come genesi dell'anemia. Il coinvolgimento renale si può manifestare in forma di insufficienza renale con o senza oligoanuria e proteinuria anche in un range nefrosico. Nel $20 \%$ dei casi, si osservano, inoltre, manifestazioni extra-renali soprattutto a carico del sistema nervoso centrale (10\%). Sono riportati: infarto miocardico (3\%), lesioni ischemiche periferiche e, in una percentuale di casi che arriva fino al $5 \%$, una disfunzione multiorgano dovuta a una TMA diffusa che si presenta con coinvolgimento neurologico, ischemia miocardica, emorragia polmonare con insufficienza respiratoria, pancreatite, coinvolgimento epatico e sanguinamento gastrointestinale $(2,3)$.

Spesso i sintomi d'esordio sono sfumati e la triade classica di presentazione può non essere completa. In uno studio retrospettivo, Sallée et al hanno identificato, in due differenti coorti, al momento della diagnosi, la presenza di livelli normali di piastrine nel $13 \%$ dei pazienti (4). Tutto ciò può porta- 
re a una maggiore difficoltà nell'interpretazione del quadro e a un ritardo nel processo diagnostico.

In presenza di un quadro di TMA, è necessario effettuare una diagnosi differenziale tra i due principali quadri clinici responsabili (TTP e SEU) mediante il dosaggio dell'attività dell'ADAMTS 13. L'ADAMTS 13 è una proteasi responsabile del clivaggio del fattore di von Willebrand, il cui deficit è alla base dello sviluppo della TTP. In presenza, quindi, di una sua attività normale possiamo escludere la presenza di TTP. II passo diagnostico successivo per poter formulare una diagnosi di aSEU è l'esclusione di tutte le patologie sistemiche e/o dei farmaci che possano causare una microangiopatia trombotica con conseguente quadro secondario di SEU (Tab. I). Solo I'indagine genetica, tuttavia, confermerà l'ipotesi clinica. È bene ricordare che, benché le analisi genetiche siano consigliate in tutti i pazienti al primo episodio di aSEU, esse non sono necessarie per la diagnosi della malattia in fase acuta ma giocano un ruolo importante nella gestione a lungo termine del paziente.

TABELLA I - Forme secondarie di microangiopatia trombotica

\begin{tabular}{|c|c|}
\hline Gravidanza & $\begin{array}{l}\text { HELLP } \\
\text { Post-partum }\end{array}$ \\
\hline Malattie sistemiche & $\begin{array}{l}\text { Lupus Eritematoso Sistemico } \\
\text { Sindrome da anticorpi anti-fosfolipidi } \\
\text { Sclerodermia }\end{array}$ \\
\hline Infezioni & $\begin{array}{l}\text { Influenza H1N1 } \\
\text { Epatite A o C } \\
\text { HIV } \\
\text { Citomegalovirus, Epstein-Barr virus } \\
\text { Streptococcus Pneumoniae } \\
\text { Human Parvovirus }\end{array}$ \\
\hline Neoplasie & $\begin{array}{l}\text { Mammella, ovaio } \\
\text { Stomaco, colon } \\
\text { Polmone } \\
\text { Pancreas } \\
\text { Linfoma }\end{array}$ \\
\hline Trapianto & $\begin{array}{l}\text { Trapianto di midollo } \\
\text { Trapianto d'organo }\end{array}$ \\
\hline \multicolumn{2}{|l|}{ Ipertensione maligna } \\
\hline \multicolumn{2}{|l|}{ Deficit di cobalamina } \\
\hline Farmaci & $\begin{array}{l}\text { Inibitori della calcineurina } \\
\text { Mitomicina } \\
\text { Chinino } \\
\text { Cisplatino } \\
\text { Vincristina } \\
\text { Contraccettivi orali } \\
\text { Interferone } \\
\text { Clopidogrel } \\
\text { Eroina, cocaina }\end{array}$ \\
\hline
\end{tabular}

\section{Patogenesi}

Alla base dello sviluppo della aSEU vi è un'attivazione non controllata della via alternativa del complemento. Si assiste, infatti, a una sregolata produzione di C3 convertasi, che induce un eccessivo clivaggio di C5 che, a sua volta, porta a un'incontrollata produzione di complesso di attacco della membrana, normalmente deputato alla citolisi di agenti patogeni. Nel soggetto con aSEU, l'endotelio non è in grado di difendersi efficacemente dall'azione di questo complesso. Il danno endoteliale innesca l'attivazione e il reclutamento di piastrine con la formazione di trombi a livello del microcircolo renale e di altri organi (soprattutto sistema nervoso centrale) (5).

L'ipotesi patogenetica della aSEU è di tipo multi-step. Mutazioni o polimorfismi a carico dei geni che codificano per le proteine regolatrici del complemento sono stati identificati in circa il $60 \%$ dei pazienti e rendono il soggetto suscettibile allo sviluppo della malattia. Possono essere presenti più mutazioni in eterozigosi nello stesso soggetto. Per motivi non noti, inoltre, la penetranza del fenotipo è incompleta (circa il 50\%). Sono state identificate mutazioni a carico dei geni che codificano per il fattore $\mathrm{H}$ del complemento (CFH), il fattore I del complemento (CFI), la proteina cofattore di membrana (MCP), il fattore $B$ del complemento, la componente $\mathrm{C} 3$ del complemento e la trombomodulina (THBD). Sono, infine, state identificate forme di aSEU associate allo sviluppo di anticorpi diretti contro il CFH.

In presenza di questo substrato favorente agiscono eventi trigger, infezioni soprattutto a carico delle alte vie aeree o del tratto gastroenterico, in grado di innescare il processo patologico e di portare allo sviluppo della malattia.

\section{Terapia}

Fino al 2011, il trattamento di prima scelta per la aSEU era la terapia con plasma, in forma di infusione o plasmaferesi. La dimostrazione della sua efficacia si basa soprattutto su un'expert opinion, non essendo mai stati effettuati trial prospettici. Come riportato nel registro italiano, la risposta alla terapia e, quindi, la prognosi della malattia sono influenzate dal tipo di mutazione genetica riscontrata nel paziente (1). Nella pratica clinica, la terapia con plasma deve essere iniziata empiricamente il prima possibile, entro 24 ore dall'esordio, e proseguita, con stretto monitoraggio degli indici di attività della malattia (livelli di emoglobina, LDH, aptoglobina, conta piastrinica e funzione renale), almeno fino a che non siano disponibili i risultati dell'attività di ADAMTS 13. Una volta esclusa la presenza di TTP, è possibile l'introduzione di una terapia aSEU-specifica.

Dal 2011, è stato approvato dalla Food and Drug Administration (USA) e dalla European Medicine Agency (EMA) I'utilizzo terapeutico nella aSEU di un agente anti-complemento chiamato Eculizumab. L'Eculizumab è un anticorpo monoclonale ricombinante umanizzato diretto contro la frazione C5 del complemento. La sua azione inibisce il clivaggio di C5 impedendo la formazione del complesso di attacco del- 
la membrana, elemento chiave nello sviluppo del processo microangiopatico alla base della aSEU. Come tutti i farmaci inibitori della cascata complementare, l'Eculizumab espone il paziente a un aumentato rischio di infezioni meningococciche. Pertanto, tutti i pazienti che richiedono una terapia con Eculizumab devono essere sottoposti a una vaccinazione antimeningococcica due settimane prima del suo inizio. Se le condizioni cliniche impongono un inizio tempestivo della terapia, è necessario associare un'adeguata profilassi antibiotica (6).

L'efficacia del farmaco è stata dimostrata in due trial di fase 2. II primo includeva 17 pazienti con malattia in fase acuta e TMA in progressione nonostante terapia con plasma. II secondo includeva 20 pazienti con storia di malattia più lunga, in remissione dipendente dalla terapia con plasma e portatori di insufficienza renale. L'Eculizumab si è dimostrato efficace nell'indurre una normalizzazione dei parametri ematologici della malattia ( $88 \%$ nel trial 1 e $90 \%$ nel trial 2), nel consentire una sospensione della terapia con plasma $(88 \%$ nel trial 1 e $100 \%$ nel trial 2) e nel miglioramento della funzione renale. La terapia dialitica è stata sospesa in 4 dei 5 pazienti che ne avevano necessità prima dell'inizio del trattamento con Eculizumab. In entrambi i trial, infine, l'inizio precoce della terapia con Eculizumab si associava a un outcome renale migliore (7).

Nonostante l'introduzione della terapia con Eculizumab abbia notevolmente migliorato la prognosi dei pazienti con aSEU, il suo utilizzo, in virtù anche degli elevati costi, non è scevro da interrogativi ancora aperti, il più importante dei quali è la durata della terapia con Eculizumab, vista anche l'assenza di informazioni sui rischi a lungo termine legati alla sua somministrazione. Non esistono, inoltre, test specifici utilizzabili nella pratica clinica in grado di monitorare tale terapia e che consentano di sviluppare uno schema terapeutico ad personam. Ardissino et al hanno effettuato un tentativo di sospensione della terapia con Eculizumab in 10 pazienti in remissione, monitorati al domicilio attraverso stick urinari per identificare precocemente l'eventuale comparsa di microematuria. Solo 3 di questi pazienti hanno sviluppato una recidiva di aSEU, suggerendo che sia possibile, in presenza di una remissione stabile di malattia, provare a sospendere la terapia con Eculizumab mantenendo una stretta sorveglianza (8).

Sarebbe, infine, auspicabile lo sviluppo di biomarcatori in grado di monitorare il danno endoteliale che consentano di valutare l'andamento della malattia e la previsione dell'eventuale comparsa di recidiva.

\section{Conclusioni}

Le conoscenze acquisite negli ultimi anni sul coinvolgimento del sistema del complemento nello sviluppo della aSEU hanno reso possibile una migliore comprensione dell'eziopatogenesi della malattia e hanno gettato le basi per l'utilizzo dell'Eculizumab per il suo trattamento. Nonostante sia una patologia rara e di non facile identificazione, è necessario tenerla sempre presente nell'iter diagnostico delle microangiopatie trombotiche e cercare di identificarla rapidamente, vista la disponibilità di una terapia efficace che deve essere iniziata il più precocemente possibile. Permangono, tuttavia, questioni non risolte come le possibili cause dello sviluppo della malattia nei pazienti nei quali non è stato possibile identificare mutazioni e lo sviluppo di schemi terapeutici ottimali.

\section{Disclosures}

Financial support: No financial support was received for this submission.

Conflict of interest: The authors have no conflict of interest.

\section{Bibliografia}

1. Noris M, Caprioli J, Bressin E, et al. Relative role of genetic complement abnormalities in sporadic and familial aHUS and their impact on clinical phenotype. Clin J Am Soc Nephrol. 2010; 5:1844-59.

2. Sellier-Leclerc AL, Fremeux-Bacchi V, Macher MA, et al. Differential impact of complement mutations on clinical characteristics in atypical hemolytic uremic syndrome. J Am Soc Nephrol. 2007;18:2392-400.

3. Nester CM, Barbour T, Rodriquez de Cordoba S, et al. Atypical aHUS: state of the art. Mol Immunol. 2015;67:31-42.

4. Sallée $M$, Ismail K, Fakhouri F, et al. Thrombocytopenia is not mandatory to diagnose haemolytic and uremic syndrome. BMC Nephrol. 2013;14:3.

5. Loirat C, Frémeaux-Bacchi V. Atypical hemolytic uremic syndrome. Orphanet J Rare Dis. 2011;6:60.

6. Zuber J, Fakhouri F, Roumenina LT, Loirat C, Frémeux-Bacchi V. Use of Eculizumab for atypical haemolytic uraemic syndorme and C3 glomerulophaties. Nat Rev Nephrol. 2012;8:643-57.

7. Legendre $\mathrm{CM}$, Licht $\mathrm{C}$, Muus $\mathrm{P}$, et al. Terminal Complement Inhibitor Eculizumab in Atypical Hemolytic-Uremic Syndrome. N Engl J Med. 2013;368:2169-81.

8. Ardissino G, Testa S, Possenti I. Discontinuation of Eculizumab maintenance treatment for atypical hemolytic uremic syndrome: a report of 10 cases. Am J Kidney Dis. 2014;64(4):633-7. 